\title{
The modelled occurrence of non-thermal plasma in the ionospheric F-region and the possible consequences for ion outflows into the magnetosphere
}

Article

Published Version

Lockwood, M. and Fuller-Rowell, T. J. (1987) The modelled occurrence of non-thermal plasma in the ionospheric F-region and the possible consequences for ion outflows into the magnetosphere. Geophysical Research Letters, 14 (4). pp. 371-374. ISSN 0094-8276 doi:

https://doi.org/10.1029/GL014i004p00371 Available at https://centaur.reading.ac.uk/38899/

It is advisable to refer to the publisher's version if you intend to cite from the work. See Guidance on citing.

Published version at: http://dx.doi.org/10.1029/GL014i004p00371

To link to this article DOI: http://dx.doi.org/10.1029/GL014i004p00371

Publisher: American Geophysical Union

All outputs in CentAUR are protected by Intellectual Property Rights law, including copyright law. Copyright and IPR is retained by the creators or other copyright holders. Terms and conditions for use of this material are defined in the End User Agreement. 


\section{www.reading.ac.uk/centaur}

\section{CentAUR}

Central Archive at the University of Reading

Reading's research outputs online 
THE MODELLED OCCURRENCE OF NON-THERMAL PLASMA IN THE IONOSPHERIC F-REGION AND THE POSSIBLE CONSEQUENCES FOR ION OUTFLOWS INTO THE MAGNETOSPHERE

\title{
M. Lockwood
}

Rutherford Appleton Laboratory

\author{
T.J. Fuller-Rowell
}

University College, London

\begin{abstract}
A global, time-dependent, threedimensional, coupled ionosphere-thermosphere model is used to predict the spatial distribution of non-thermal plasma in the F-layer. It is shown that, even for steady-state conditions with $\mathrm{Kp}$ as low as 3 , the difference between the ion and neutral velocities often exceeds the neutral thermal speed by a factor, $D^{\prime}$, which can be as large as 4. Theoretically, highly non-Maxwellian, and probabiy toroidal, ion velocity distributions are expected when $D^{\prime}$ exceeds about 1.5 . The lack of response of the neutral winds to sunward ion drifts in the dawn sector of the auroral oval cause this to be the region most likely to contain toroidal distributions. The maximum in $\mathrm{D}^{2}$ is found in the throat region of the convection pattern, where the strong neutral winds of the afternoon sector meet the eastward ion flows of the morning sector. These predictions are of interest, not only to radar scientists searching for non-thermal ionospheric plasma, but also as one possible explanation of the initial heating and upward flows of ions in the cleft ion fountain and nightside auroral oval, both of which are a major source of plasma for the magnetosphere.
\end{abstract}

\section{Introduction}

Recent observations by the EISCAT radar have revealed non-thermal F-region plasma in the dayside auroral oval (Lockwood et ai., 1987b). The non-Maxwellian ion velocity distributions are identified during bursts of rapid plasma flow, which are consistent with the expected ionospheric signature of flux transfer events at the magnetopause (Todd et al., 1986). The short-lived nature of these events allows the non-Maxwellian effects to be identified by the changes in the scattered power and spectrum width, as well as in the shape of the spectrum. Previous1y, St-Maurice et al (1976) had observed non-Maxwellian distributions using a retarding potential analyser, but the spatial-temporal ambiguity of these satellite observations means that it is not known if these were induced by steady or transient ion flows. The same EISCAT experiment as was used to detect the flow bursts (UK-POLAR) has observed spectra with the characteristic shape for non-thermal plasma over periods of more than an hour when the ion flow was rapid but steady. However, spectral noise and sampling prevent the unambiguous identification of "steady-state" non-Maxwellian distributions if spectral shape is the only

Copyright 1987 by the American Geophysical Union.

Paper number 6L6496.

0094-8276/87/006L-6496\$03.00 available evidence (as arguments relating to the time constants for changes in the plasma density, neutral composition and winds, as used for the flow bursts by Lockwood et al, cannot be applied). Nevertheless, such non-thermal plasmas, and/or associated micro-instabilities, are expected for the observed magnitudes of steady ion drifts and this paper presents an initial set of predictions of when and where they will occur and discusses possible implications for bulk upward flows of ionospheric plasma.

\section{Conditions For Non-Maxwellian Plasma}

St-Maurice and Schunk (1979) have presented a review of predicted ion velocity distributions for a variety of models for the ion-neutral collision process. The simplest is the "relaxation mode1", for which the ion velocity after a collision is taken to equal that of the neutrai atom/molecule prior to the collision. This is, therefore, a good approximation to the charge exchange interaction. St-Maurice and Schunk have demonstrated that, for this model, the mean of the modulus of the ion velocity is roughly equal to the the ion-neutral relative velocity, $\left|\bar{v}_{i}-\bar{v}_{n}\right|$, and that the spread about that mean is given by the thermal speed of the neutral gas. The distribution of the fieldperpendicular velocities shows a minimum at the origin (i.e. is toroidal) if this mean exceeds the spread, i.e. if the ratio $D^{\prime}$ exceeds unity:

$$
D^{\prime}=\left|\bar{v}_{i}-\bar{v}_{n}\right| /\left(2 k_{B} T_{n} / m_{n}\right)^{1 / 2},
$$

where $T_{n}$ and $m_{n}$ are the temperature and mass of the neutral thermospheric gas and $k_{B}$ is Boltzmann's constant. This conclusion can be applied when the frequency of ion-neutral collisions is much smaller than the ion gyrofrequency but still exceeds that of fon-ion collisions. In practice this is valid for an altitude range of about $200-400 \mathrm{~km}$.

In fact; departures from a bi-Maxwellian form are apparent for the relaxation model when $D^{\prime}>$ 0.75 . Evaluation of the distribution function for more realistic models of ion-neutral collisions is much more complex. Barakat et al. (1983) used Monte-Carlo simulation techniques to allow for both charge exchange and polarization elastic scatter. The latter interaction can scatter perpendicular ion velocity into the parallel direction and hence tends to destroy the toroidal form. The important conclusion reached by these authors is that toroids should still form, but the ion drift required would be higher than predicted by the relaxation model. They stess that their results are only "semi-quantitative", however, from their table 2 we conclude $D^{\prime}>1.5$ 

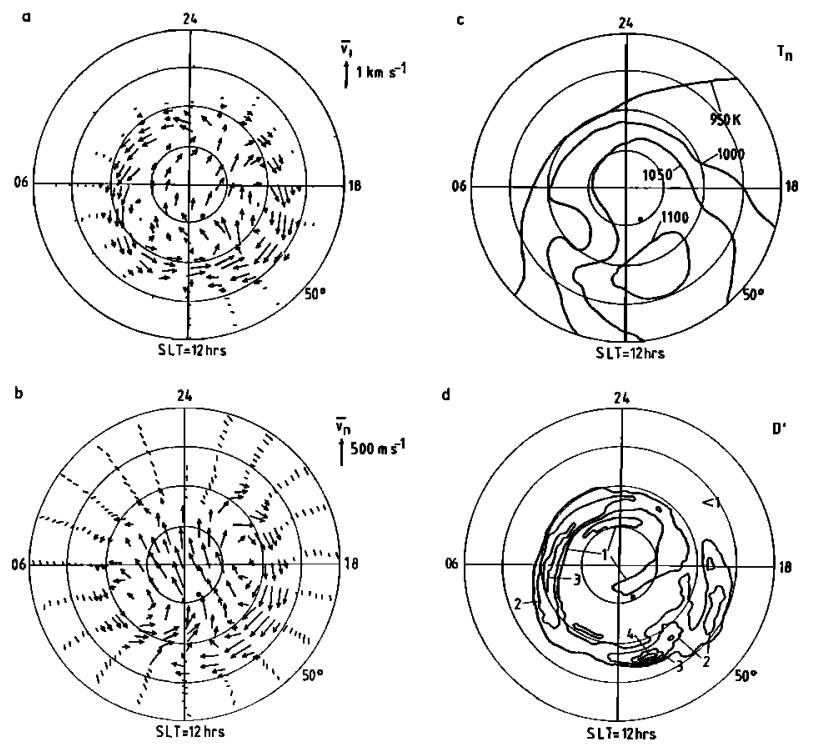

Fig. 1. Model values for the northern hemisphere at $300 \mathrm{~km}$ for $18 \mathrm{UT}$ and December solstice with $\mathrm{F} 10.7=165, \mathrm{Kp}=3$ and $\mathrm{B}<0$ and shown as $a$ function of Solar Local Time (SLT) and geographic latitude over the range $50-90^{\circ}$. The position of the magnetic pole is denoted by a dot. (a) Ion velocity vectors, $\bar{v}_{1}$; (b) equilibrium neutral winds, $\bar{\nabla}_{n} ;$ (c) neutral temperature, $T_{n}$; and (d) the ratio $D^{\prime}$.

is required and $D^{\prime}=2$ certainly gives a more clearly defined torus than $D^{\prime}=1$ for the relaxation model.

Lastly, we note that the results of Barakat et al do not allow for the effects of instabilities driven by, but also tending to destroy, the toroidal distribution. The instability with the lowest threshold has been predicted to be the Post-Rosenbluth.

\section{Modelled distribution of $\mathrm{D}^{\prime}$}

A global, time dependent, three-dimensional model of the coupled ionosphere-thermosphere system has been developed by University College, London and the University of Sheffield (FullerRowell et al., 1987). The model self-consistently evaluates the plasma densities and neutral wind, temperature and composition for an assumed pattern of the convection electric field and auroral precipitation. For a given location, UT, magnetic activity and time of year, all the parameters in equation (1) can be evaluated for the convection pattern adopted. The initial results presented here are for a negative $B_{y}$ component of the interplanetary magnetic field, simulated by using the Heppner and Maynard (1983) A-2 model of convection equipotentials in the northern hemisphere and the B-2 model in the southern hemisphere. In both cases the total cross-cap potential is $76 \mathrm{kV}$, which drives ion velocities of up to about $1 \mathrm{~km} \mathrm{~s}^{-1}$ in the auroral oval. The important features can be illustrated by considering one UT ( 18 hours) at December solstice. The auroral activity level used is only moderate and equivalent to a Kp 3 , and an F10.7 index value of 165 was adopted (near sunspot maximum). A fuller parametric study of the effects of these parameters will be presented at a later date. The spatial distributions of $D^{p}$ for both hemispheres was evaluated for "steadystate" conditions, i.e. the electric field patterns have been stable for a sufficiently long period to allow the neutral winds to have reached equilibrium with them in a diurnal sense. Full details of the model and of the computational procedure are given by Fuller-Rowell et a1 (1987).

Figures 1 and 2 show inputs to and outputs from the model at 18 UT. In all cases, the plots are in geographic latitude - solar local time (SLT) polar dial format, with local noon at the bottom, dawn to the left and dusk to the right, and the position of the magnetic pole is marked with a dot. The geographic frame is used in preference to a magnetic one as the neutral winds and temperature are computed in that frame and it is the most useful for identifying the times when ground-based radars such as EISCAT should be able to detect non-thermal plasma. The plots cover the geographic latitude range $50-90^{\circ}$ and are for $a$ constant pressure level corresponding to an altitude close to $300 \mathrm{~km}$. Figure 1 is for the northern (winter) hemisphere and shows (a) the ion drifts, $\bar{v}_{i}$; (b) the neutral winds, $\bar{v}_{n}$; (c) the neutral exospheric temperature, $T_{n}$ and (d) the ratio $D^{\prime}$. Figure $1(a)$ shows the two-celled convection pattern projected into this reference frame at this UT. The "throat" of the pattern, where plasma flows into the polar cap, occurs near 12 SLT and the Harang discontinuity is in the region 19-23 SLT. In figure 1 (b), the neutral winds, $\bar{\nabla}_{n}$, are shown, but note the factor of 2 scaling difference between parts (a) and (b) of figure 1. One obvious difference between the ion and neutral flow patterns is that the dawn and dusk cells are of roughly equal strength and size for the ion flow pattern, whereas the sunward neutral winds are relatively strong in the dusk cell but are weak in the dawn cell. Since this dawn-dusk asymmetry strongly influences the results for $D^{\prime}$, it is worth reminding ourselves of its origin (Fuller-Rowell et al., 1984). In the dusk sector, the coriolis and centrifugal accelerations of a packet of neutral air tend to cancel and its motion is largely westward, air which remains within the region of strong auroral ion drifts aquires a large westward velocity. Conversely, in the dawn cell these two accelerations add and move the packet equatorward and out of the region of large ion drifts, preventing it from aquiring a large momentum from the ions. This dawn-dusk asymmetry has been observed in mean auroral winds from a variety of experiments (Killeen et al., 1986). The neutral temperature is given in figure $1(\mathrm{c})$ and shows the expected peak values near noon due to solar and

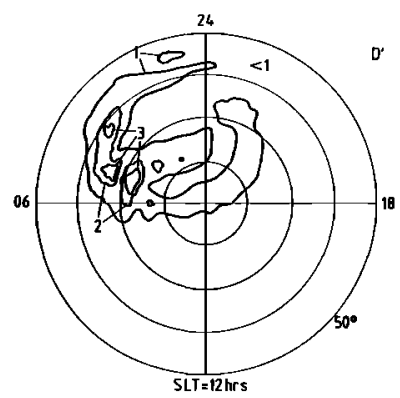

Fig. 2. The ratio $D^{\prime}$ in the southern hemisphere shown for the same conditions and format as figure 1 . 
cusp heating and lower values on the nightside. Note that in computing $\mathrm{D}^{\prime}$, the mean mass of the neutral gas was used for $m_{n}$. This did not vary significantly from 16 amu in winter, but what variation there was tended to reduce the effect of the variations in $T_{n}$

The resulting map of $D^{\prime}$ for the northern hemisphere is shown on figure 1 (d). Generally, $D^{\prime}$ only exceeded unity within the auroral oval, the only exception to this being a part of the polar cap (where $D^{\prime}$ is less than 1.5). Everywhere in the nightside, dusk cell (SLT=18-23) auroral oval $D^{\prime}$ remains near to unity, whereas large values (>3) are found in the nightside, dawn sector oval (SLT $=0-6$ ). The largest values of $D^{\prime}$ are found near the zero potential contour of the dayside convection pattern (SLT near 13 hours) and parts (a) and (b) of figure 1 show that in this region the strong westward winds established in the afternoon sector meet the eastward ion flows of the dawn convection cell, giving $D^{\prime}$ values as large as 4 despite the elevated $T_{n}$ values on the dayside.

Figure 2 shows the spatial distribution of $D^{\prime}$ for the southern (summer) hemisphere under the same conditions. In this case, the zero potential contour is at earlier SLT (and MLT - a feature of the B-2 convection model) and again this region gives the largest values for $D^{\prime}(43)$. However, in figure 2 the peak value of $D^{\prime}$ occurs at slightly lower SLT than the zero potential contour (i.e. on the dawn cell) whereas it is at slightly greater SLT in figure 1(d) (on the dusk celi). Hence the peak appears on the more crescent-shaped cell of the asymmetric convection pattern for general $B_{y}$. Values are generally lower in figure 2 than for the winter case because of the higher $T_{n}$. The dawn-dusk asymmetry on the nightside is even more marked than in figure $1(d)$.

Discussion of Possible Relevance to Ion Outflows Into the Magnetosphere

The cleft ionosphere is known to be a strong and continuous ion source for the magnetosphere (see review by Lockwood, 1987). "Upwelling ions" are observed carrying upward heat fluxes and showing the effects of both perpendicular and parallel ion heating in the "cleft ion fountain" effect. These flows not only contain the heavy ionosheric ions $\mathrm{O}^{+}$and $\mathrm{N}^{+}$, but molecular ions detected in the polar magnetosphere have been traced back to this source. This implies a heating mechanism operating at F-region altitudes where such massive ions are expected and indeed thermal upwellings have been reported in the dayside auroral oval from the HILAT and DE2 satellites near $800 \mathrm{~km}$ (see Lockwood, 1987).

Figures 1 and 2 demonstrate that highly non-Maxwellian plasmas are expected in the dayside auroral F-region, even for quiet, nonvarying conditions. The dawn-dusk asymmetry in $D^{\prime}$ is similar to one which be seen in the occurrence of large field-aligned flows in the topside ionosphere, as reported by Lockwood (1983) and in heavy ion outflows into the magnetosphere (figure 4 of Yau et al., 1986). We conclude that nonthermal plasmas should be present in those regions where large upflows of heavy ions have been observed.

There are two ways in which the effects of non-isotropic ion velocity distributions on upward field-aligned flows can be evaluated. Firstly, Schunk et al. (1975) have employed an additional field-aligned stress tensor term in the hydrodynamic equations to allow for an anisotropic distribution. For fixed (no-flow) boundary conditions at the top of the ionosphere, these authors showed how the plasma density profile is effected by the additional term. However, they did not compute how the outflow would be altered in the absence of this boundary condition. In addition, in a kinetic model, a toroidal ion velocity distribution would give rise to an additional nett upforce due to the gradient-B mirror force. Work is currently underway to compute the ion velocity distribution function for a given $D^{\prime}$ and hence quantify the effective ion temperatures and the additional force and flows. One major problem is that the collisional model employed and any microinstabilities can greatly effect the results obtained. However, initial results for the relaxation collision model indicate that $D^{\prime}>3$ could give average upward mirror forces which are a significant fraction (up to $\rightarrow 0.3$ ) of the gravitational force on the ions (Suvanto, private communication).

Lastly, we note that similar mechanisms for driving ion outflows have previously been suggested in relation to transversely accelerated ions (TAIs - see references given by Lockwood, 1982). In both cases the mirror force acts to overcome gravity and the charge exchange barrier presented by neutral hydrogen. However, TAIs are non-Maxwellian in that they form a hot tail to the distribution function, whereas the mechanism suggested here is via ion distributions the core of which has been perturbed from a Maxwellian.

\section{Discussion and Conclusions}

The model results show that $D^{\prime}$ values as high as 3-4 should result from high latitude plasma convection, even for steady convection with $\mathrm{Kp}$ as low as 3 . The precise thresholds for driving toroidal and non-Maxwellian plasmas are not known, but are not expected to exceed $D^{\prime}=2$ and Lockwood et al. (1987b) observed them when $D^{\prime}$ exceeded unity. The spatial distribution of $D^{\prime}$ shows a maximum in the area where large ion heating and upflows are required to explain upwelling ion events and the cleft ion fountain and is high in the nightside regions where upflows have been observed.

The high values of $D^{\prime}$ in the cleft region also give an indication of the likely response of the ion flows to changes in the convection pattern. Large-scale expansions of the polar cap with simultaneous ion outflows have recently been reported (Lockwood et al, 1987a). If the neutral wind pattern had reached diurnal equilibrium with the ion flows before the expansion, the increase in cross-cap potential would initially drive high $D^{\prime}$ values everywhere in the high latitude region, but the expansion of the convection pattern would cause particularly high values in the annulus of newly-opened flux tubes where antisunward ion flows would initially meet sunward neutral winds. The increased $D^{\prime}$ values may enhance ion outflows which have been predicted in response to the transient Joule heating increase without allowance for any non-thermal effects (Gombosi and Killeen, 1987). Fuller-Rowell and Rees (1981) have shown that the wind pattern responds most 
rapidly in the afternoon sector, hence $D^{\prime}$ would decay in this sector first. Eventually, a pattern similar to those in figures 1 (d) and 2 would emerge, for the expanded auroral oval.

It is useful to compare this scenario with observations from the DE2 spacecraft (Killeen et al.,1984). These authors reported "hot-spots" of velocity difference between ions and neutrals were sometimes restricted to the dawn auroral oval and sometimes were seen in the entire polar region and other parts of the oval. We would suggest that the former cases are consistent with the steady-state predictions of $D^{\prime}$ presented here and that the latter are due to time-varying electric field patterns.

Lastly, we note that the coupled model makes no allowance for the effects of the high $D^{\prime}$ values on the plasma density. In general high $D^{\prime}$ should deplete the plasma, via the suggested ion upflows and by increasing the ion-neutral loss reaction rates (see St-Maurice and Schunk, 1979). This would act to further decouple the ion and neutral flows and hence, by equation (1) would tend to increase $D^{\prime}$.

Acknowledgements. The authors are grateful to all the many scientists at UCL and Sheffield University who have assisted in the development of the model.

\section{References}

Barakat, A.R., R.W. Schunk, and J.-P. St-Maurice, Monte-Carlo calculations of the O+ velocity distribution in the auroral ionosphere, J. Geophys. Res., 88, 3237-3241, 1983.

Fuller-Rowe11, T.J. and D. Rees, A three dimensional, time-dependent simulation of the global dynamical response of the thermosphere to a geomagnetic storm, J. Atmos. Terr. Phys., 43, 701-722, 1981.

Fuller-Rowe11, T.J., S. Quegan, D. Rees, G.J. Bailey, and R.J. Moffett, The effect of realistic conductivities on the high-latitude neutral thermospheric circulation, Planet. Space Sci., 32, 469-480, 1984.

Fuller-Rowe11, T.J., D. Rees, S. Quegan, G.J. Bailey, and R.J. Moffett, Interactions between neutral thermospheric composition and the polar ionosphere using a coupled ionosphere-thermosphere model. J. Geophys. Res., in press, 1987.

Gombosi, T.I and T.L. Killeen, Effects of thermospheric motions on the polar wind: a time dependent numerical study, J. Geophys. Res., in press, 1987.

Heppner, J.P. and N.C. Maynard, High latitude electric field models, Chapman conference on magnetospheric currents, AGU, Irvington, Va., April 5-8, 1983.

Killeen, T.L., P.B. Hays, G.R. Gordon, R.A. Heelis, W.B. Hanson, N.W. Spencer, and L.H.
Brace, Ion-Neutral Coupling in the highlatitude F-region:evaluation of ion heating terms from Dynamics Explorer 2, J. Geophys. Res., 89, 7495-7508, 1984.

Killeen, T.L., R.G. Roble, R.W. Smith, N.W. Spencer, J. Meriwether, D.Rees, G. Hernandez, P.B. Hays, L.I. Cogger, D.P. Sipler, M.A. Biondi, and C.A. Tepley, Mean neutral circulation in the winter polar F-region, $\mathrm{J}$. Geophys. Res., 91, 1633-1649, 1986.

Lockwood, M., Thermal ion flows in the topside auroral ionosphere and the effects of low-altitude transverse acceleration, Planet. Space Sci., 30, 595-609, 1982.

Lockwood, M., Low-energy ion flows into the magnetosphere, Adv. in Space Res., 6 (3), $63-77,1987$.

Lockwood, M., A.P. van Eyken, B.J.I. Bromage, J.H. Waite, Jr., T.E. Moore, and J.R. Doupnik, Low-energy ion outflows from the ionosphere during a major cap expansion - evidence for equatorward motion of inverted-V structures, Adv. in Space Research, 6 (3), 93-101, 1987a.

Lockwood, M., B.J.I. Bromage, R.B. Horne, J.-P. St-Maurice, D.M. Willis, and S.W.H. Cowley, Non-Maxwellian ion velocity distributions observed using EISCAT, Geophys. Res. Lett., 14, $111-114,1987 \mathrm{~b}$.

Schunk, R.W., W.J. Raitt, and P.M. Banks, Effect of high electric fields on the daytime high latitude $E$ and $F$ regions, J. Geophys. Res., 80, 3121-3130, 1975 .

St-Maurice, J.-P., W.B. Hanson, and J.C.G. Walker, Retarding potential analyser measurement of the effect of ion-neutral collisions on the ion velocity distribution in the auroral ionosphere, J. Geophys. Res., 81, 5438-5446, 1976.

St-Maurice, J.-P. and R.W. Schunk, Ion velocity distributions in the high-latitude ionosphere, Rev. Geophys. Space Phys,, 17, 99-134, 1979.

Todd, H., B.J.I. Bromage, S.W.H. Cowley, M. Lockwood, A.P. van Eyken, and D.M. Willis, EISCAT observations of bursts of rapid flow in the dayside high latitude ionosphere, Geophys. Res. Lett., 13, 909-912, 1986.

Yau, A.W., E.G. Shelley, and W.K. Peterson, Accelerated auroral and polar cap ions: outflow at $D E-1$ altitudes, in "Ion acceleration in the ionosphere and magnetosphere "ed. T. Chang, AGU monograph 38, 72-76, 1986.

T.J. Fuller-Rowe11, Dept. Physics and Astronomy, University College London, Gower Street, London WC1E 6BT, UK

M. Lockwood, Rutherford Appleton Laboratory, Chilton Didcot, Oxfordshire OX11 OQX, UK

(Received December 31, 1986; revised February 5, 1987; accepted February 20, 1987.) 